\title{
Secondary-side-only Control for High Efficiency and Desired Power with Two Converters in Wireless Power Transfer Systems
}

\author{
Giorgio Lovison*a) $^{*}$ Student Member, Daita Kobayashi* Non-member \\ Motoki Sato ${ }^{* *} \quad$ Member, Takehiro Imura ${ }^{* * *}$ Member \\ Yoichi Hori* Fellow
}

(Manuscript received Jan. 12, 2017, revised June 20, 2017)

\begin{abstract}
In recent years, wireless power transfer has been a popular research topic because it allows both static and dynamic charging without using cables, thus easing the process for the user. However, achieving high efficiency and desired power corresponding to the load at any time is important. Communication between sides is often used, but sometimes its operation is not guaranteed because of harsh environmental conditions or malfunctioning. This paper addresses this problem by proposing a novel yet simple control strategy. It consists of simultaneous regulation of both power and efficiency by the secondary side alone, without communication with the primary side. When the primary side is not manipulated, the power is controlled by an AC/DC converter by using a two-mode control. On the other hand, the efficiency is controlled by a DC/DC converter by adjusting the DC link voltage to a reference value related to the maximum wireless transmitting efficiency. In this paper, the control concept as well as the design are provided. The proposed control shows a good trade-off by achieving power regulation capability at the cost of slight reduction in efficiency when compared to the currently existing state-of-the-art control.
\end{abstract}

Keywords: wireless power transfer, power control, efficiency control, secondary side

\section{Introduction}

Wireless power transfer (WPT) technology recently has become appealing for household, industrial and automotive applications because it simplifies the powering process and eliminates potential dangers for the user. WPT by magnetic resonant coupling achieves high efficiency and is capable of transmitting high power ${ }^{(1)}$. In particular, it grants robustness against misalignment, increased air gap and higher transmission efficiency when compared to conventional induction method $^{(2)(3)}$. There are different types of WPT tuning by magnetic resonant coupling, depending on the compensation topology ${ }^{(4)}$ : the series-series (SS) compensation has received much interest because it allows high power to flow on the secondary side with high efficiency. These characteristics are favorable for applications such as Electric Vehicles (EVs), as abundant literature confirms ${ }^{(4)-(8)}$. Currently, static WPT (i.e. when the coils are not moving) is available on the market; however, developing dynamic WPT (i.e. when the coils' reciprocal position is changing) is necessary as a way to reduce

a) Correspondence to: Giorgio Lovison. E-mail: lovison.giorgio14 @ ae.k.u-tokyo.ac.jp

* The University of Tokyo, Graduate School of Frontier Sciences, Advanced Energy Department

5-1-5, Kashiwanoha, Kashiwa, Chiba 277-8561, Japan

** Toyodenki Seizo K.K., Engineering Research Division 3-8, Fukuura, Kanazawa-ku, Yokohama, Kanagawa 236-0004, Japan

*** The University of Tokyo, Graduate School of Engineering, Electrical Engineering and Information Systems Department 7-3-1, Hongo, Bunkyo-ku, Tokyo 113-8656, Japan both battery weight and range anxiety ${ }^{(8)}$; recently, some trial units are been experimented upon and achieved promising results ${ }^{(9)-(12)}$. The same concept can be applied as well to household appliances such as laptops and mobile phones.

In WPT systems, converter control is generally oriented into power control and efficiency control. Previous papers dealt with power control on primary side ${ }^{(13)(14)}$, efficiency control on secondary side ${ }^{(15)-(17)}$ or power and efficiency control by using both sides ${ }^{(18)-(20)}$. If efficiency control was performed on the secondary side, the power had to be regulated on the primary side and vice versa, as until now it has been impossible to manipulate them both on the same side without using complex phase-shift controls ${ }^{(21)}$. In particular, primary side voltage regulation is a popular choice for power flow control and may be a necessity in case of particular applications such as medical implants to limit the secondary side heating ${ }^{(13)}$. Moreover, communication between sides is also needed for feedback signal and eventually coil detection. However, if communication fails, the primary side is not properly controlled anymore. Thus, from a control point of view, a novel method applied only on the secondary side, able to transfer at the same time the desired power with high efficiency without communicating with the primary side is desirable.

Previous literature encompassing converter control without communication between sides dealt with secondary side efficiency maximization ${ }^{(21)}$ as well as primary side maximum efficiency tracking ${ }^{(22)}$ and charging time control ${ }^{(23)}$. In ${ }^{(21)}$, a complex phase-shift control regulating the imaginary part of the load impedance is devised for non-resonance operation. The studies in ${ }^{(22)}$ and ${ }^{(23)}$ use a three level voltage waveform 
generated in the primary side and successively regulated by a closed loop control by a converter on the secondary side. These controls require precise timing and therefore advanced boards. On the other hand, two-mode control is a simple regulation method implemented by a Half Active Rectifier (HAR), as presented in many papers such as ${ }^{(20)(24)}$. In these studies, the overall system efficiency when the power is on the kilowatt order is over 0.88 , including the losses generated while shorting the HAR input terminals. By considering a WPT system with two converters on the secondary side, it is possible to fulfill the requirements for simultaneous power and efficiency control only by manipulating those converter and without commanding the primary side. However, unlike the study in ${ }^{(21)}$, this is achievable in a much simpler way by using the HAR to control the average power and the DC/DC converter to control the transmission efficiency. This method is based on using two different switching frequencies for the converters and can be applied to any WPT system. This is actually an extension of the concept proposed in ${ }^{(25)}$, with a much clearer controller design and the inclusion of a mutual inductance estimation method to use in a dynamic charging scenario. The experimental results show that the proposed control is able to retain an efficiency comparable to the stateof-the-art while regulating the power to the desired value in both static and dynamic charging scenarios.

This paper is organized into seven sections. Section 2 introduces the case of study. Section 3 describes the power and efficiency characteristics of WPT while Section 4 explains the proposed control concept. Section 5 illustrates the control design and the setting of the operation points. Section 6 presents the experimental results of the static charging scenario. Section 7 points out the challenges in a dynamic charging scenario, and shows the proposed control experimental results. Section 8 finally draws the conclusions from the results.

\section{Case of Study}

2.1 Fundamental Characteristics of WPT This study focuses on the secondary side of a WPT system with series-series (SS) compensation, as shown in the equivalent circuits of Fig. 1. The resonant frequency of the system is obtained from the coil parameters, which are independent from the load and the distance between the coils. The resonant frequency $f_{\text {res }}$ and therefore the relative angular frequency $\omega$ are expressed as:

$$
\omega=2 \pi f_{\text {res }}=\frac{1}{\sqrt{L_{1} C_{1}}}=\frac{1}{\sqrt{L_{2} C_{2}}}, \cdots \cdots \cdots \cdots \cdots \cdots
$$

with $L_{1}$ and $C_{1}$ as the primary coil inductance and capacitance, respectively; similarly, $L_{2}$ and $C_{2}$ are the secondary coil inductance and capacitance. The coils' internal resistances only affect the losses and the dynamic response of the system. The mutual inductance $L_{m}$ between the primary and secondary coil depends mainly on the coil geometry and the air gap. From the circuit equations, the voltage ratio $A_{V}$ and the current ratio $A_{I}$ between the primary side and the secondary side are described as follows:

$$
A_{V}=\frac{V_{2}}{V_{1}}=j \frac{\omega L_{m} R_{L}}{R_{1}\left(R_{2}+R_{L}\right)+\left(\omega L_{m}\right)^{2}},
$$

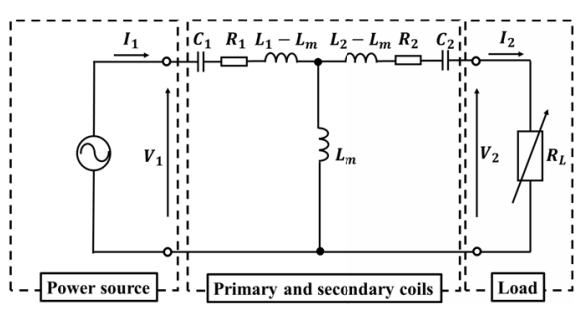

Fig. 1. Equivalent circuit of WPT with magnetic resonant coupling and SS compensation

$$
A_{I}=\frac{I_{2}}{I_{1}}=j \frac{\omega L_{m}}{R_{2}+R_{L}}
$$

where $I_{1}$, and $I_{2}$ are the RMS values of the primary current and the secondary current, respectively, and $R_{L}$ is the load impedance. As for $V_{1}$, it is the RMS value of the fundamental wave component of the primary inverter square wave output voltage. Similarly, $V_{2}$ is the RMS value of the fundamental wave component of the secondary AC/DC converter input voltage. The primary side coil resistance is represented by $R_{1}$, while $R_{2}$ is the one of secondary side coil; on the other hand, the equivalent load impedance is $R_{L}$. The phase shift of $\frac{\pi}{2}$ between primary and secondary parameters in (2) and (3) is expressed through $j$. The transmitting efficiency is consequently derived as following:

$$
\eta=\frac{\left(\omega L_{m}\right)^{2} R_{L}}{\left(R_{2}+R_{L}\right)\left[R_{1}\left(R_{2}+R_{L}\right)+\left(\omega L_{m}\right)^{2}\right]} . \cdots \cdots \cdots
$$

The load power can be instead obtained easily from:

$$
\begin{aligned}
P_{L} & =\frac{V_{2}^{2}}{R_{L}} \\
& =\frac{\left\|A_{V}\right\|^{2}}{R_{L}} V_{1}^{2} \\
& =\frac{\left(\omega L_{m}\right)^{2} R_{L}}{\left[R_{1}\left(R_{2}+R_{L}\right)+\left(\omega L_{m}\right)^{2}\right]^{2}} V_{1}^{2} .
\end{aligned}
$$

The influence of $R_{L}$ has been the subject of past research $^{(26)-(28)}$. In particular, the equivalent load impedance associated with maximum transmission efficiency, the one related to maximum deliverable power and the one related to the desired power are different because their operation points are different. When $R_{L}$ changes, the secondary side voltage $V_{2}$ changes accordingly. In case of maximum transmitting efficiency condition, the equivalent load impedance is calculated as:

$$
R_{L, \eta \max }=\sqrt{\frac{R_{2}}{R_{1}}\left(\omega L_{m}\right)^{2}+R_{2}^{2}}
$$

Its related secondary side voltage $V_{2, \text { max }}$ is given by:

$$
V_{2, \eta \text { max }}=\sqrt{\frac{R_{2}}{R_{1}}} \frac{\omega L_{m}}{\sqrt{R_{1} R_{2}+\left(\omega L_{m}\right)^{2}}+\sqrt{R_{1} R_{2}}} V_{1} \cdots(7)
$$

The voltage in (7) is a RMS value. The load power $P_{L}$ is active power and related to the fundamental wave components of AC parameters. Therefore, in this paper, the focus will be on the fundamental wave components of $V_{1}$ and $V_{2}$. In addition, the secondary side current $I_{2}$ is assumed to be a sinusoidal wave, in phase with $V_{2}$. 


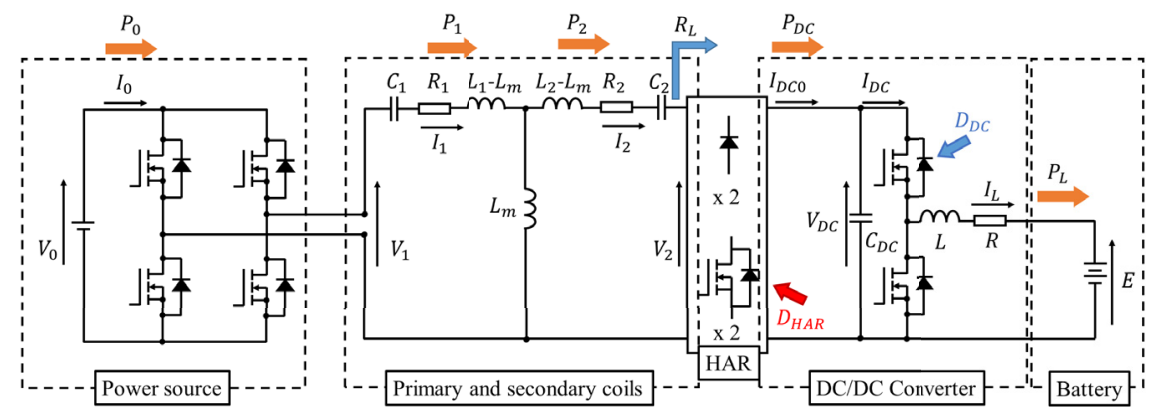

Fig. 2. Reference circuit of WPT system
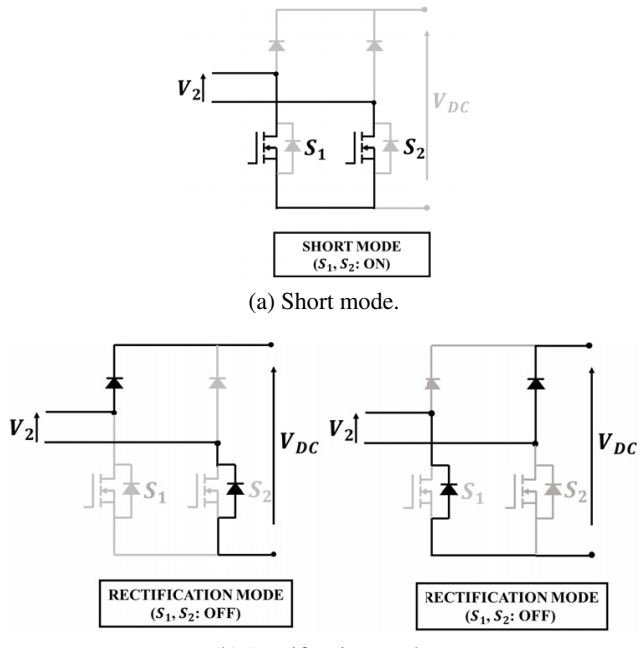

(b) Rectification mode.

Fig. 3. HAR operation modes

2.2 Topology In Fig. 2 is shown the reference circuit. The coils' inductances and the compensating capacitors are designed according to (1). The primary side includes a DC voltage source and an inverter, where in the secondary side there are a HAR, a DC/DC converter and the battery load. The battery is considered not optimized to receive ripple current, therefore the capacitance $C_{D C}$ and inductor $L$ are chosen in such a way that the current $I_{D C 0}$ is smoothened enough before reaching the battery.

The HAR is an AC/DC converter whose topology resembles the bridgeless PFC circuit ${ }^{(24)}$. In the high side there are two diodes, but in the low side there are two active devices. Its operation is divided into two modes, as shown in Fig. 3:

- Short mode: both the low side devices $S_{1}$ and $S_{2}$ are turned ON. In so doing, the coil terminal are shortcircuited and no current flows to the secondary DC bus. The DC link voltage $V_{D C}$ consequently decreases as the smoothing capacitor $C_{D C}$ supplies the load. This is possible because the SS compensation topology makes the secondary side coil act like an equivalent current source, thus allowing the secondary side coil short-circuit. There is meaning in short mode because the impedance seen from the secondary side is very high, thus the primary side current $I_{1}$ becomes very low.

- Rectification mode: both the low side devices $S_{1}$ and $S_{2}$ are turned OFF. The HAR behaves like a full bridge diode rectifier and consequently the load is supplied. The DC link voltage $V_{D C}$ increases as $C_{D C}$ is charged.
By switching between these two modes, the average power can be controlled. The HAR operation limits secondary side heating but increases the electromagnetic interference $(\mathrm{EMI})^{(24)}$. Finally, the duty cycle expresses the ratio between short mode and switching period, as written in (8):

$$
0<D_{H A R}=\frac{t_{\text {short }}}{t_{\text {short }}+t_{\text {rect }}}=t_{\text {short }} f_{\text {HAR }}<1,
$$

with $f_{H A R}$ as the HAR switching frequency.

\section{Proposed Control Concept}

In this paper, a novel control combination carried out entirely on the secondary side of a WPT system is proposed. The HAR and the DC/DC converter operate independently from the primary side inverter, whose duty cycle is fixed. The secondary side is not synchronized with the primary side and do not benefit from any kind of communication device (e.g. Bluetooth). The simultaneous regulation comprises the average power control by the HAR and the efficiency control through DC link voltage regulation via DC/DC converter. The proposed control method can be adopted for any power level.

In the conventional maximum efficiency control from secondary side, the operation point is fixed. For a given $R_{L}$, there are a unique secondary power $P_{2}$ and a unique DC-to-DC efficiency $\eta_{D C}$. Hence, when the load impedance seen from the secondary coil is equal to $R_{L, \text { max }}$, the efficiency is $\eta_{\max }$ and the power is $P_{L, \eta \max }$. This will not change unless the mutual inductance $L_{m}$ or the primary side voltage $V_{1}$ varies. In the proposed control, the operation point is the same; however, the switching between short mode and rectification mode allows manipulating the average power $P_{D C}$. Hence, it is possible to send a different power value while maintaining nearly the same transmitting efficiency.

The control concept can be visually explained by Fig. 4. In rectification mode, the RMS value of secondary side voltage is unchanged because the DC/DC converter controls $V_{D C}$ to be equal to $V_{D C, \eta \max }$ according to (9):

$$
V_{D C, \eta \max }=\frac{\pi}{2 \sqrt{2}} V_{2, \eta \max } .
$$

The RMS value of secondary current $I_{2}$ during rectification mode can be calculated by rearranging (2) and (3) and is given by ${ }^{(29)}$ :

$$
I_{2}=\frac{\omega L_{m} V_{1}-R_{1} V_{2}}{R_{1} R_{2}+\left(\omega L_{m}\right)^{2}} .
$$

However, given the short mode, the HAR output current $I_{D C 0}$ 


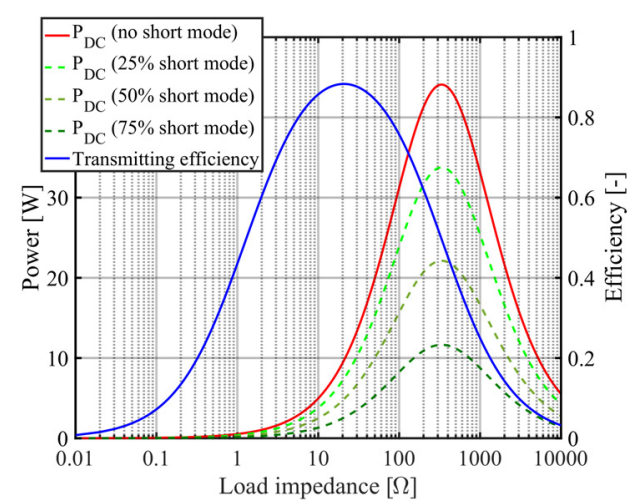

(a) Power and efficiency curves for the parameters in Table 1.

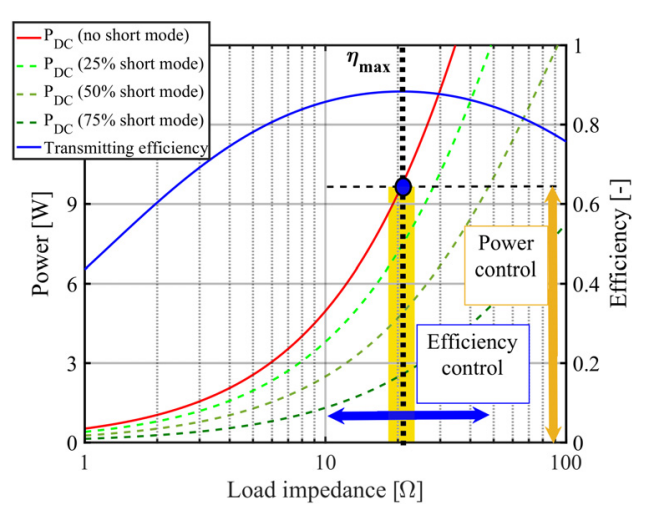

(b) Enlargement of power and efficiency curves with operation ranges.

Fig. 4. Proposed control concept

can be rewritten by considering Fourier series and its value is equal to:

$$
I_{D C 0}= \begin{cases}\frac{2 \sqrt{2}}{\pi} I_{2} & \text { (Rectification mode) } \\ 0 & \text { (Short mode }) .\end{cases}
$$

Since $I_{D C 0}$ 's value depends on the operation mode of the HAR, the average value of (11) over the HAR switching period can be derived as follows:

$$
\bar{I}_{D C 0}=\frac{2 \sqrt{2}}{\pi} I_{2}\left(1-D_{H A R}\right) .
$$

The power $\bar{P}_{D C}$ is the parameter under control and is calculated via multiplication of (12) by (9). Therefore, $\bar{P}_{D C}$ is the averaged value of $P_{D C}$.

It should be noted that, during short mode, the smoothing capacitor $C_{D C}$ provides the power to the load. Consequently, $P_{L}$ is not intermittent but a continuous value and the load is always supplied with a high transmitting efficiency. The proposed control is highlighted by the transparent area in Fig. 4(b). For the experimental evaluation of the DC-toDC efficiency, the DC powers in both primary and secondary side are taken into account. With reference to Fig. $2, P_{0}$ is the primary side DC power and $P_{D C}$ is the secondary side one. However, since $P_{D C}$ is an averaged value which includes short mode time, the DC-to-DC efficiency will be slightly lower. In fact, during rectification mode the DC-to-DC efficiency is maximum, while during short mode it will drop. In this latter case, there are losses in the secondary side coil and the primary side circuit due to the circulating current. However, the reflected impedance to the primary side becomes very high, causing $I_{1}$ to be very low. Hence, considering the HAR switching period, the short mode losses $P_{\text {short }}$ consist mainly in conduction loss determined by $I_{2}$ and $R_{2}$ and the on-resistance of active devices $S_{1}$ and $S_{2}$. On the contrary, the rectification mode losses $P_{\text {rect }}$ include both conduction and switching losses in both sides. In other words, $P_{\text {rect }}$ is much bigger than $P_{\text {short }}$. Thus, the DC-to-DC efficiency reduction due to averaging is limited and is proportional to $R_{2}$ and the active devices' on resistance. In fact, both $P_{0}$ and $P_{D C}$ change along $D_{H A R}$, so that their ratio is unvaried and the only difference is the addition of short mode losses.

As for the waveforms of the proposed control, they are shown in Fig. 5. The HAR input voltage $V_{2}$ becomes close to zero during short mode, while the HAR input current $I_{2}$ is

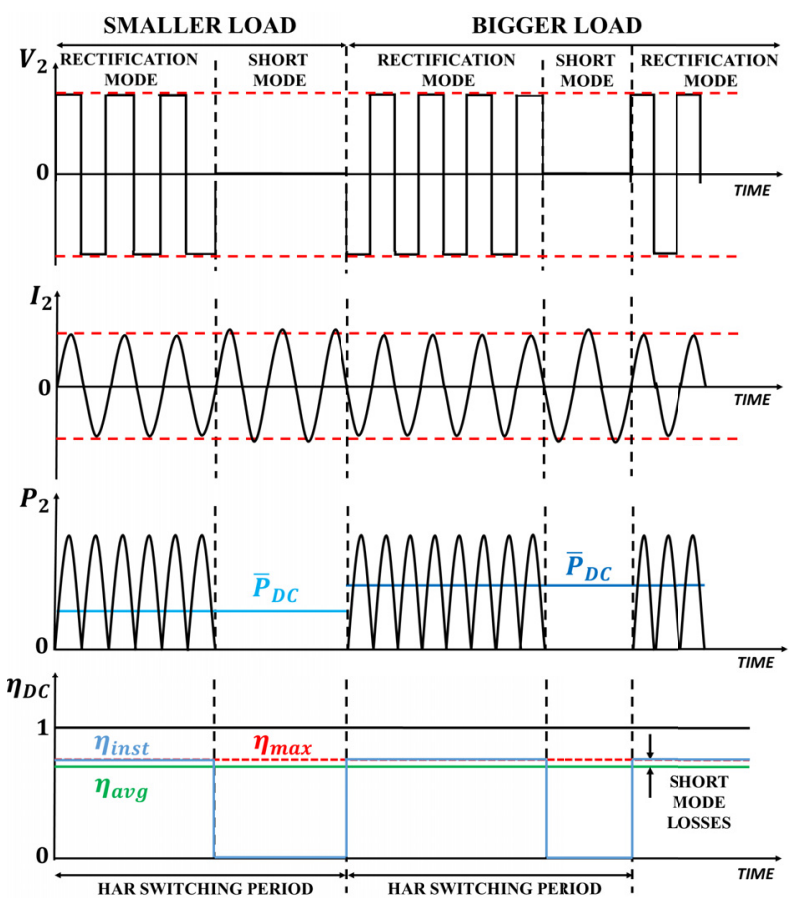

Fig. 5. Parameters waveforms

slightly higher. The input power $P_{2}$ then changes accordingly to the previous waveforms; its average value $P_{D C}$ depends on short mode duration. As for efficiency, the instantaneous value $\eta_{\text {inst }}$ is equal to the maximum value $\eta_{\text {max }}$ in rectification mode, while in short mode it is equal to zero. When calculating the efficiency over the switching period, the averaged value is constant even if the instantaneous value changes according to the operation mode.

\section{Controller Design}

In the previous chapter, the proposed control method has been explained. In order to proceed with the controller design, the following two conditions are assumed:

(1) The primary side source voltage $V_{0}$, the resonant frequency $f_{\text {res }}$, the system operation frequency $f$ and the mutual inductance $L_{m}$ are given and fixed.

(2) The HAR switching frequency is at least one order of magnitude lower than the DC/DC converter switching frequency and their values are fixed.

The first assumption means that the primary side has a fixed 


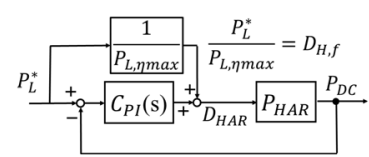

(a) HAR.

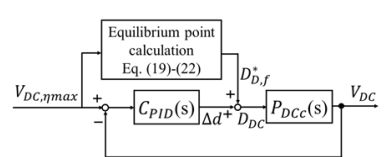

(b) DC/DC converter.
Fig. 6. Block diagram of controllers

operation point that sets the limits for maximum load power and the maximum transmitting efficiency achievable. If the $V_{0}$ changes, the proposed control will still operate but with a lower performance.

The second assumption prevents operation conflicts due to the two different time constants. Switching frequency modulation is not considered in this paper.

The block diagram of both HAR and DC/DC controllers are shown in Fig. 6. Each controller is composed by a feedforward part and a feedback part. The feedback part of both the controllers is designed with the pole placement method.

4.1 HAR Controller The HAR controller actually controls the current because the DC/DC converter regulates the secondary DC voltage $V_{D C}$. Considering (11) and (12), the HAR plant can be derived easily and it is given by:

$$
P_{D C}=\left\{\begin{array}{ll}
P_{2} & (\text { Rectification mode }) \\
0 & (\text { Short mode })
\end{array} .\right.
$$

Then, by the same averaging expression over the switching time used previously, (13) becomes:

$$
P_{H A R}=\bar{P}_{D C}=P_{2}\left(1-D_{H A R}\right)
$$

The linearized plant model of the HAR is simple, consequently a PI controller $C_{P I}(s)$ is deemed sufficient.

In order to set the equilibrium point, it is necessary to know the value of the transmitted power at maximum efficiency condition. First, by substituting (7) in (10), the secondary current in maximum efficiency condition is obtained as following:

$$
I_{2, \eta \max }=\frac{\omega L_{m} V_{1}-R_{1} V_{2, \eta \max }}{R_{1} R_{2}+\left(\omega L_{m}\right)^{2}} . \cdots \ldots \ldots \ldots \ldots \ldots
$$

Then, by simply multiplying (7) to (15), the transmitted power at maximum efficiency condition $P_{L, \text { max }}$ is calculated and it is given by:

$$
P_{L, \eta \max }=V_{2, \eta \max } I_{2, \eta \max }
$$

The feedforward part of the HAR duty $D_{H, f}$ is then determined as the ratio between the desired power $P_{L}^{*}$ and (16):

$$
D_{H, f}=\frac{P_{L}^{*}}{P_{L, \eta \max }} .
$$

Clearly, $P_{L}^{*}$ must be smaller than $P_{L, \eta \text { max }}$, otherwise the proposed control will not operate as expected. If higher power is needed, the solution simply consists in increasing the primary side voltage $V_{1}$ and recalculating the parameters.

4.2 DC/DC Converter Controller In this case of study, the DC/DC converter is a buck converter. Past research described thoroughly how to model the converter by the state space averaging ${ }^{(27)(30)}$. Thus, in this paper the modeling process will be omitted. Since the DC/DC converter is a nonlinear system, it is necessary to linearize the converter plant and obtain (18) for control design. The linearized converter plant $P_{D C c}$ is a transfer function from $\Delta d$ to the DC link voltage $V_{D C}$, given by:

$$
\begin{aligned}
& P_{D C c}(s)=\frac{\Delta V_{D C}}{\Delta d}=\frac{b_{1} s+b_{0}}{s^{2}+a_{1} s+a_{0}} \cdots \cdots \\
& a_{1}=\frac{R}{L}+\frac{8}{\pi^{2}} \frac{R_{1}\left(1-D_{H A R}\right)}{C_{D C}\left[R_{1} R_{2}+\left(\omega L_{m}\right)^{2}\right]} \\
& a_{0}=\frac{1}{L C_{D C}}\left[D_{D, f}+\frac{8}{\pi^{2}} \frac{R R_{1}\left(1-D_{H A R}\right)}{R_{1} R_{2}+\left(\omega L_{m}\right)^{2}}\right] \\
& b_{1}=-\frac{I_{L}}{C_{D C}}, \quad b_{0}=-\frac{R I_{L}+D_{D, f} V_{D C}}{L C_{D C}}
\end{aligned}
$$

where $R$ and $L$ are the DC/DC converter resistance and inductance, respectively; $D_{D, f}$ is the feedforward part composing the high side device duty cycle and $I_{L}$ is the load current. The transfer function is a second order system, therefore it is desirable to use a PID controller $C_{P I D}(s)$. In the controller, a low pass filter is cascaded with the derivative term to reduce the high frequency noise. The aim of the control is matching $V_{D C}$ with the maximum efficiency voltage, whose expression is derived by (9). The equilibrium value in (19) and (20) satisfy the state space model of DC/DC converter and are given as follows:

$$
\begin{aligned}
& V_{D C}^{*}=\frac{E D_{D, f}^{*}+R I_{D C}^{*}}{D_{D, f}^{*}{ }^{2}}=V_{D C, \eta \max }, \cdots \ldots \ldots \ldots \ldots \ldots \ldots \ldots \ldots \ldots \ldots \ldots \ldots \ldots \ldots \ldots \ldots \ldots
\end{aligned}
$$

where $V_{D C}^{*}, I_{L}^{*}, I_{D C}^{*}$ and $D_{D, f}^{*}$ are the equilibrium point values of $V_{D C}, I_{L}, I_{D C}$ and $D_{d, f}$, respectively. From here, it is possible to compute the remaining equilibrium points. The DC/DC converter input voltage reference $I_{D C}^{*}$ can be derived from (9), (11), and (18):

$$
I_{D C}^{*}=\frac{8}{\pi^{2}} \frac{\omega L_{m} V_{0}-R_{1} V_{D C}^{*}}{R_{1} R_{2}+\left(\omega L_{m}\right)^{2}}\left(1-D_{H A R}\right)
$$

Finally, the feedforward part of the DC/DC converter duty $D_{D, f}^{*}$ is computed from (19) and (21) as follows:

$$
D_{D, f}^{*}=\frac{E+\sqrt{E^{2}-4 R V_{D C}^{*} I_{D C}^{*}}}{2 V_{D C}^{*}}
$$

\section{Static Charging Scenario}

In order to verify the effectiveness of the proposed method, experiments have been performed in a static scenario. In this scenario the coil position is fixed, therefore the mutual inductance $L_{m}$ is constant.

The experimental setup is shown in Fig. 7. It includes a DC generator (TAKASAGO ZX-400LA), the primary side inverter, the primary and secondary coils, the HAR, the DC/DC converter and the load battery. Both the HAR and the DC/DC converter use silicon MOSFETs (IRFB3607) as active devices. The control is carried out with a DSP board (Myway PE-PRO/F28335A). In each side there are one voltage and one current sensor: in the primary side the inverter input voltage $V_{0}$ and current $I_{0}$ (and consequently $P_{0}$ ) are measured; the secondary side sensors measure the HAR output current 


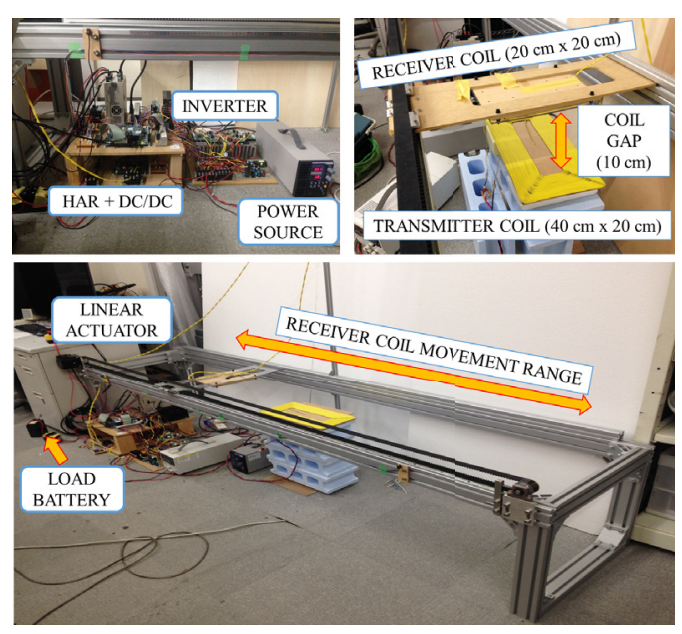

Fig. 7. Experimental setup

$I_{D C 0}$ and voltage $V_{D C}$ (and consequently $P_{D C}$ ). The reason for using only DC sensors is to reduce the installation cost. The receiving coil is mounted on a stage and it is possible to move it via a linear actuator. The experiment parameters are reported in Table 1. The proposed control is compared with the secondary side efficiency control in order to prove that the desired power is delivered with near maximum efficiency. The comparison is carried out by experiments. According to the laws of the authors' country ${ }^{(31)(32)}$, the power level must be kept under $50 \mathrm{~W}$ in a system using high frequency. Thus, the experiments are performed on low power, but the concept can be applied to any power level.

The experimental results for the secondary side efficiency control and the proposed one are shown in Fig. 8 and Fig. 9, respectively. In the first case, the secondary side control only maximizes $\eta_{D C}$. Thus, the power is not manipulated at all and $\eta_{D C}$ is the maximum achievable efficiency for the system. In the other case, a desired power of $3 \mathrm{~W}$ is considered; then, halfway in the experiment, the power reference has a step change up to $6 \mathrm{~W}$. This means that the HAR duty cycle $D_{H A R}$ has a step change. This variation is introduced to prove the proposed control robustness. In both the conventional state-of-the-art efficiency control and the proposed one, the efficiency maximization is achieved with the equivalent load impedance optimization method, as explained in Section 2 and Section 4; this method is sound and it has been adopted in previous literature as ${ }^{(20)-(22)}$ and ${ }^{(25)-(30)}$. In the figures, the instantaneous values are indicated by thin lighter lines while averaged values are represented by thicker dark lines.

In Fig. 8(a) and Fig. 9(a), the DC link voltage $V_{D C}$ is controlled to match the reference value, represented by the black dashed line. In particular, Fig. 9(a) shows that the voltage control of the proposed method is resistant to power steps.

The DC power $P_{D C}$ is shown in Fig. 8(b) and Fig. 9(b). In Fig. 8(b) the power is not controlled; on the other hand, Fig. 9(b) shows that $P_{D C}$ is successfully controlled. In fact, the instantaneous power fluctuates due to the switching between the operation modes. Here, the red line represents $P_{0}$, the blue line is $P_{D C}$ and the black dashed line is the secondary side power reference.

The load power $P_{L}$ is shown in Fig. 8(c) and Fig. 9(c). With the secondary side efficiency control, it is impossible
Table 1. System parameters

\begin{tabular}{c|c}
\hline \hline Parameter & Value \\
\hline \hline Primary side DC voltage source $V_{0}$ & $18 \mathrm{~V}$ \\
\hline Primary side coil resistance $R_{1}$ & $1.83 \Omega$ \\
\hline Secondary side coil resistance $R_{2}$ & $1.683 \Omega$ \\
\hline Primary side coil inductance $L_{1}$ & $417.8 \mu \mathrm{H}$ \\
\hline Secondary side coil inductance $L_{2}$ & $208.3 \mu \mathrm{H}$ \\
\hline Primary side coil capacitance $C_{1}$ & $6.03 \mathrm{nF}$ \\
\hline Secondary side coil capacitance $C_{2}$ & $12.15 \mathrm{nF}$ \\
\hline Operation frequency $f$ & $100 \mathrm{kHz}$ \\
\hline Mutual inductance $L_{m}$ (best alignment) & $37.9 \mu \mathrm{H}$ \\
\hline Coil gap (best alignment) & $100 \mathrm{~mm}$ \\
\hline Smoothing capacitor $C_{D C}$ & $1000 \mu F$ \\
\hline DC/DC converter resistance $R$ & $0.2 \Omega$ \\
\hline DC/DC converter inductance $L$ & $1000 \mu \mathrm{H}$ \\
\hline Load battery voltage $E$ & $6 \mathrm{~V}$ \\
\hline HAR switching frequency $f_{H A R}$ & $0.5 \mathrm{kHz}$ \\
\hline DC/DC converter switching frequency $f_{D C}$ & $10 \mathrm{kHz}$ \\
\hline \hline
\end{tabular}

to change the power; however, as shown in Fig. 9(c), the proposed control successfully regulates the power to the desired value.

Finally, the DC-to-DC efficiency $\eta_{D C}$ is shown in Fig. 8(d) and Fig. $9(\mathrm{~d})$. The instantaneous value is obtained from the ratio between $P_{D C}$ and $P_{0}$. In Fig. 8(d), its value is 0.74 and it is the maximum achievable efficiency for the system; on the other hand, in Fig. 9(d) $\eta_{D C}$ follows the same pattern of the power in Fig. 9(b). However, the averaged value is 0.7 and is unvaried regardless of the step change of $D_{H A R}$, apart from the short transient occurring at the moment of the step change. As surmised in Section 3, the short mode losses depend on $R_{2}$ and the active devices' on resistance, accounting for a average DC efficiency reduction of about $4 \%$.

\section{Dynamic Charging Scenario}

Achieving highly efficient dynamic WPT will greatly widen the application range for this technology. Previous research has already been carried out with promising results ${ }^{(11)(12)}$, but in those cases the authors concentrated on the optimum coil design or the use of additional elements to solve the issues of dynamic charging. In particular, coil detection is a major issue but it is not discussed in this paper, where the focus is on the control method. Thus, the proposed control must be demonstrated in a dynamic scenario, too.

6.1 Mutual Inductance Estimation The most notable problem of dynamic WPT is the variation of mutual inductance. As it appears in nearly all expressions in this paper, it is a critical parameter for control purposes and knowing its value is necessary. Direct measurement is impossible, therefore the only feasible solution is to estimate it in real time. This paper does not focus on parameter estimation and does not provide deep analysis; however, given the similar system conditions, the method described in ${ }^{(30)}$ is adopted. The estimated $L_{m}$ is then feed to (7), whose value is continuously updated in the controllers and consequently the feedback loop can always have the desired poles.

6.2 Experimental Results In this section, too, the proposed control is compared with the secondary side 


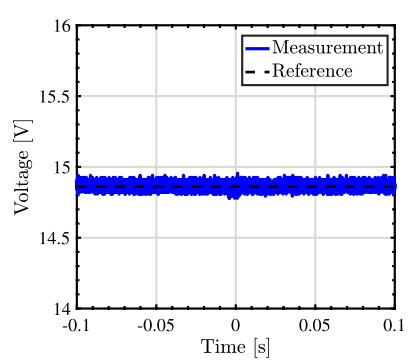

(a) DC link voltage $V_{D C}$.

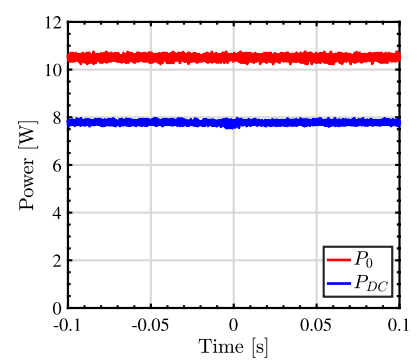

(b) Primary power $P_{0}$ and secondary power $P_{D C}$

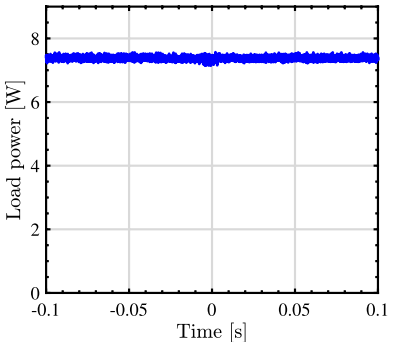

(c) Load power $P_{L}$.

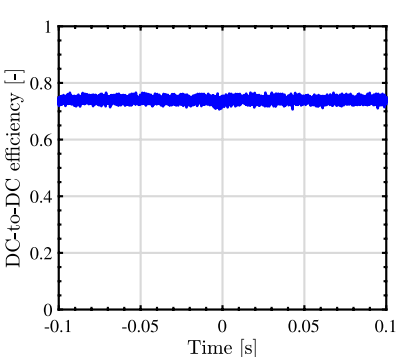

(d) DC-to-DC efficiency $\eta_{D C}$.

Fig. 8. Experimental results of secondary side efficiency control (only maximum efficiency)

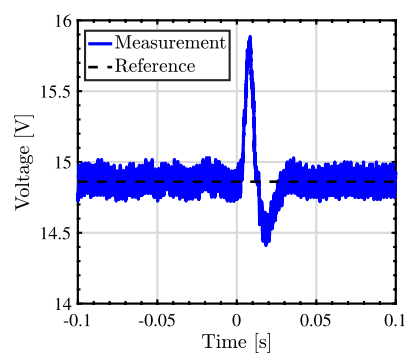

(a) DC link voltage $V_{D C}$

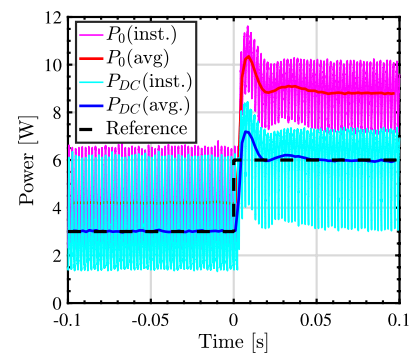

(b) Primary power $P_{0}$ and secondary power $P_{D C}$

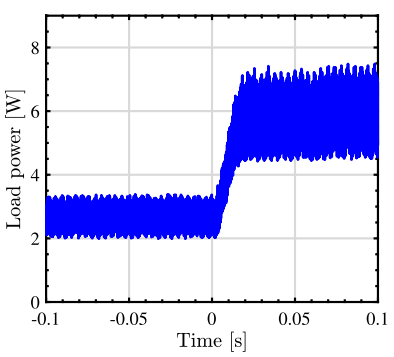

(c) Load power $P_{L}$.

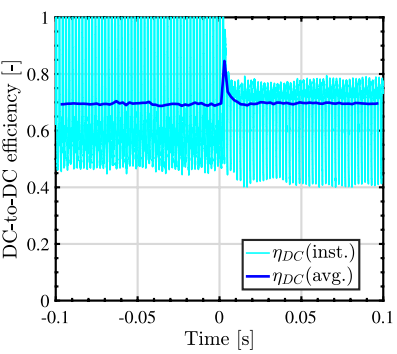

(d) DC-to-DC efficiency $\eta_{D C}$.

Fig. 9. Experimental results of proposed control (near-maximum efficiency and desired power)

efficiency control. The secondary coil is moved thanks to the stage built-in linear actuator. The stage is also equipped with an encoder to control the position and the speed of the secondary coil. In order to avoid big losses, the primary side inverter is operated only when the coils are close enough. The secondary coil speed is set to $10 \mathrm{~km} / \mathrm{h}$.

In this section, the proposed control is performed for two different power references $P_{L}^{*}$ : one is $3 \mathrm{~W}$, the other is $6 \mathrm{~W}$. The operation time goes from zero to 0.13 seconds; outside this period, the control is not operated.

The secondary side DC link voltages are shown in Fig. 10(a), Fig. 11(a) and Fig. 12(a). The variation of mutual inductance does not have a relevant impact on the maximum efficiency voltage $V_{D C, \eta \max }$. Apart from the initial rise time necessary for charging $C_{D C}$ and the final part showing minimal divergence, the controller effectively works as $V_{D C}$ matches the reference in all cases.

The primary side power $P_{0}$ and secondary side power $P_{D C}$ are shown in Fig. 10(b), Fig. 11(b) and Fig. 12(b); the red line represents $P_{0}$, the blue line is $P_{D C}$ and the black dashed line is the secondary side power reference. The power is not regulated in the case of secondary efficiency control, as shown in Fig. 10(b). On the contrary, with the proposed control $P_{D C}$ is controlled and matches the reference. This is shown in Fig. 11(b) and Fig. 12(b), where the blue line coincides with the black dashed line.

Clearly, the load power $P_{L}$ behaves accordingly to $P_{D C}$, as seen in Fig. 10(c), Fig. 11(c) and Fig. 12(c). Without control, $P_{L}$ changes along with the mutual inductance; on the other hand, when the power is controlled $P_{L}$ is unvaried, as shown in Fig. 11(c) and Fig. 12(c). A problem is the final power surge, witnessed in all three considered cases. The surge is arguably generated by the discharge of the smoothing capacitor $C_{D C}$ into the DC/DC converter inductance $L$ when the primary side inverter stops and the control is not operated.
Finally, the DC-to-DC efficiency $\eta_{D C}$ is presented in Fig. 10(d), Fig. 11(d) and Fig. 12(d). The graphic in Fig. 10(d) represents the maximum achievable efficiency of the system. In the case of proposed control, $\eta_{D C}$ behaves accordingly to the power, as seen in Fig. 11(d) and Fig. 12(d). Again, the instantaneous value presents oscillations due to the switching between the operation modes. As expected, the DC-to-DC efficiency in the proposed control is slightly lower than the one in the secondary side efficiency control due to short mode losses. However, Fig. 11(d) and Fig. 12(d) show that the control can still achieve high efficiency and simultaneously manipulate the power in a dynamic charging scenario without using any communication between sides. The HAR duty $D_{H A R}$ negatively affects the efficiency in transient low coupling condition; however, once the desired power requirement is met, the average DC efficiency reaches the nearmaximum value shown in Fig. 9(d).

\section{Conclusion}

This paper discusses a simple control regulating simultaneously the efficiency and the power flow only by secondary side for WPT systems. When communication between sides is unavailable, the proposed control is adopted to preserve power flow and efficiency. The proposed control is performed with two converters by using two different switching frequencies for power and transmitting efficiency controllers. The power is controlled by a HAR with the two-mode control and a DC/DC converter regulates the efficiency via the DC link voltage by adopting the equivalent load resistance optimization method. The HAR generates short mode losses but they have limited effect on the system. Combining the efficiency control with mutual inductance estimation, the proposed control can be applied to a dynamic charging scenario as well. Experimental results confirm the validity of the proposed method. 


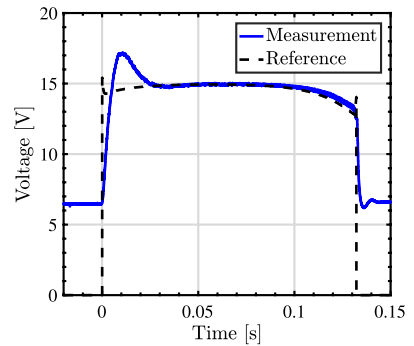

(a) DC link voltage $V_{D C}$

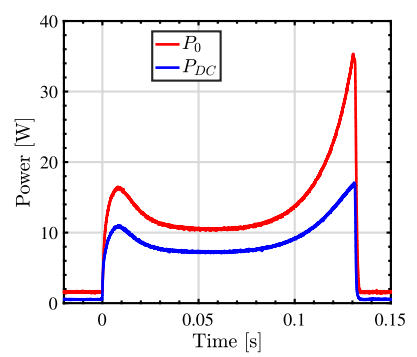

(b) Primary side power $P_{0}$ and secondary side power $P_{D C}$

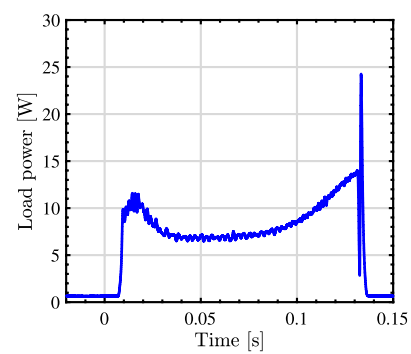

(c) Load power $P_{L}$.

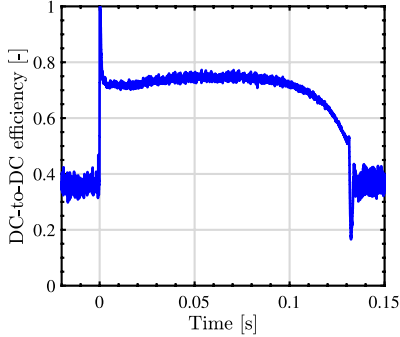

(d) DC-to-DC efficiency $\eta_{D C}$.

Fig. 10. Secondary side efficiency control ${ }^{(30)}$ when the coil speed is $10 \mathrm{~km} / \mathrm{h}$

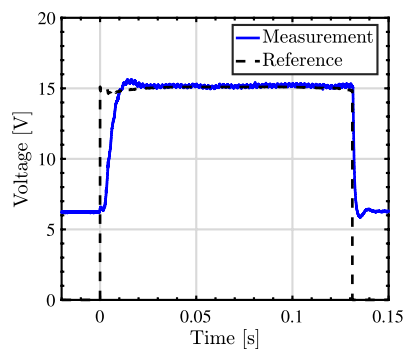

(a) DC link voltage $V_{D C}$

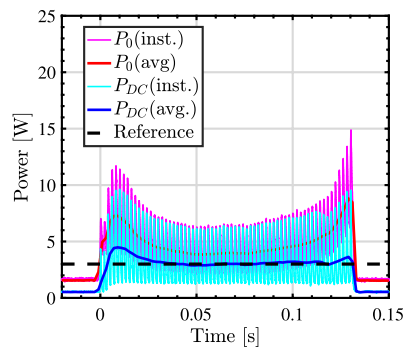

(b) Primary side power $P_{0}$ and secondary side power $P_{D C}$

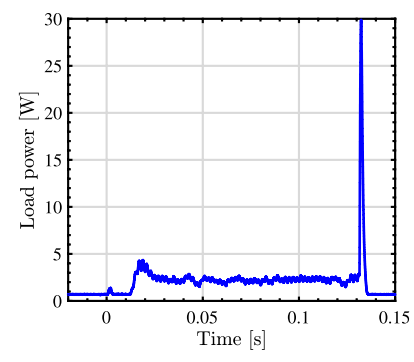

(c) Load power $P_{L}$.

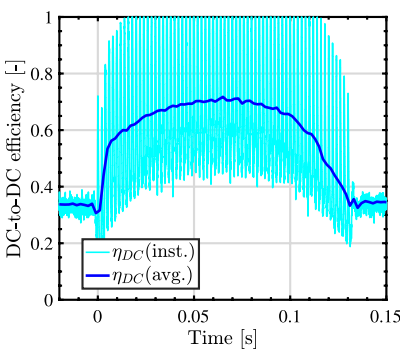

(d) DC-to-DC efficiency $\eta_{D C}$.

Fig. 11. Proposed control, power reference $3 \mathrm{~W}$ when the coil speed is $10 \mathrm{~km} / \mathrm{h}$

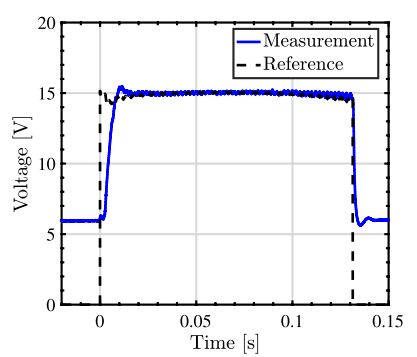

(a) DC link voltage $V_{D C}$.

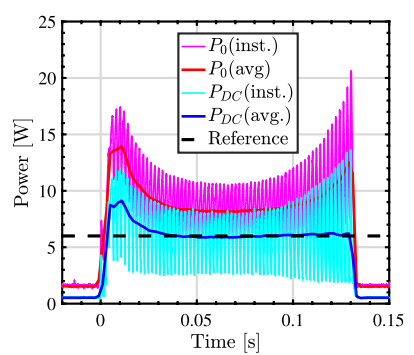

(b) Primary side power $P_{0}$ and secondary side power $P_{D C}$.

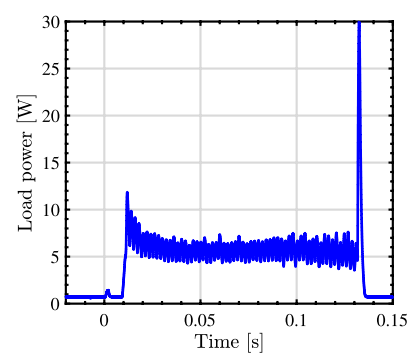

(c) Load power $P_{L}$.

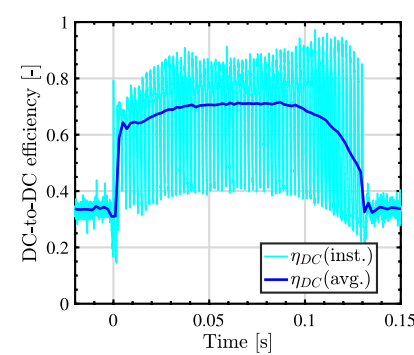

(d) DC-to-DC efficiency $\eta_{D C}$.

Fig. 12. Proposed control, power reference $6 \mathrm{~W}$ when the coil speed is $10 \mathrm{~km} / \mathrm{h}$

\section{References}

( 1 ) A. Kurs, A. Karalis, R. Moffatt, J.D. Joannopoulos, P. Fisher, and M. Soljacic: "Wireless power transfer via strongly coupled magnetic resonance", Science Express, Vol.317, No.5834, pp.83-86 (2007)

( 2 ) T. Imura, H. Okabe, and Y. Hori: "Basic experimental study on helical antennas of wireless power transfer for Electric Vehicles by using magnetic resonant coupling”, in IEEE Veh. Pow. and Prop. Conf., pp.936-940 (2009)

( 3 ) M. Kato, T. Imura, and Y. Hori: "New characteristics analysis considering transmission distance and load variation in wireless power transfer via magnetic resonant coupling", in IEEE 34th Int. Telecom. En. Conf. (INTELEC), pp.1-5 (2012)

( 4 ) S. Li and C.C. Mi: "Wireless power transfer for electric vehicle applications", IEEE Journ. of Em. and Select. Top. in Pow. El., Vol.3, No.1, pp.4-17 (2015)

( 5 ) Y. Nagatsuka, N. Ehara, Y. Kaneko, S. Abe, and T. Yasuda: "Compact contactless power transfer system for electric vehicles", in Int. Pow. El. Conf. (IPEC), pp.807-813 (2010)

( 6 ) C.S. Wang, O.H. Stielau, and G.A. Covic: "Design consideration for a contactless electric vehicle battery charger", IEEE Trans. on Ind. El., Vol.52, No.5, pp.1308-1314 (2005)

( 7 ) M. Budhia, J.T. Boys, G.A. Covic, and C. Huang: "Development of a SingleSided Flux Magnetic Coupler for Electric Vehicle IPT Charging Systems", IEEE Trans. on Ind. El., Vol.60, No.1, pp.318-328 (2013)

( 8 ) G.A. Covic and J.T. Boys: "Modern Trends in Inductive Power Transfer for Transportation Applications", IEEE Journ. of Em. and Select. Top. in Pow. El., Vol.1, No.1, pp.28-41 (2013)

( 9 ) K. Throngnumchai, A. Hanamura, Y. Naruse, and K. Takeda: "Design and evaluation of a wireless power transfer system with road embedded transmitter coils for dynamic charging of electric vehicles", in El. Veh. Symp. and Exhib. (EVS27), pp.1-10 (2013)

(10) S.W. Lee, J. Huh, C.B. Park, N.S. Choi, G.H. Cho, and C.T. Rim: "On-line electric vehicle using inductive power transfer system", in IEEE En. Conv. Cong. and Expo. (ECCE), pp.1598-1601 (2010)

(11) J. Huh, S.W. Lee, W.Y. Lee, G.H. Cho, and C.T. Rim: "Narrow-width inductive power transfer system for on-line electrical vehicles", IEEE Trans. on Pow. El., Vol.26, No.12, pp.3666-3679 (2011)

(12) J.M. Miller, O.C. Onar, C. White, S. Campbell, C. Coomer, L. Seiber R. Sepe, and A. Steyerl: "Demonstrating Dynamic Wireless Charging of an Electric Vehicle: The Benefit of Electrochemical Capacitor Smoothing", IEEE Pow. El. Mag., Vol.1, No.1, pp.12-24 (2014)

(13) H.L. Li, A.P. Hu, G.A. Covic, and C. Tang: "A new primary power regulation method for contactless power transfer", in IEEE Int. Conf. on Ind. Tech. (ICIT), pp.1-5 (2009)

(14) J.M. Miller, C.P. White, O.C. Onar, and P.M. Ryan: "Grid side regulation of wireless power charging of plug-in electric vehicles", in IEEE En. Conv. Cong. and Expo. (ECCE), pp.261-268 (2012)

(15) M. Fu, C. Ma, and X. Zhu: "A cascaded boost-buck converter for high efficiency wireless power transfer systems", IEEE Trans. on Ind. Informat., Vol.10, No.3, pp.1972-1980 (2014)

(16) K. Kusaka and J. Itoh: "Reduction of Reflected Power Loss in an AC-DC Converter for Wireless Power Transfer Systems", IEEJ Journ. of Ind. Appl., Vol.2, No.4, pp.195-203 (2013)

(17) H. Ishihara, F. Moritsuka, H. Kudo, S. Obayashi, T. Itakura, A. Matsushita, H. Mochikawa, and S. Otaka: "A voltage ratio-based efficiency control method for $3 \mathrm{~kW}$ wireless power transmission", in IEEE Appl. Pow. El. Conf. and Expo. (APEC), pp.1312-1316 (2014) 
(18) H.H. Wu, A. Gilchrist, K.D. Sealy, and D. Bronson: "A high efficiency $5 \mathrm{~kW}$ inductive charger for EVs using dual side control", IEEE Trans. on Ind. Informat., Vol.8, No.3, pp.585-595 (2012)

(19) T. Diekhans and R.W. De Donker: "A Dual-Side Controlled Inductive Power Transfer System Optimized for Large Coupling Factor Variations and Partial Load", IEEE Trans. on Pow. El., Vol.30, No.11, pp.6320-6328 (2015)

(20) M. Sato, G. Yamamoto, D. Gunji, T. Imura, and H. Fujimoto: "Development of Wireless In-Wheel Motor Using Magnetic Resonance Coupling”, IEEE Trans. on Pow. El., Vol.31, No.7, pp.5270-5278 (2016)

(21) A. Berger, M. Agostinelli, S. Vesti, J.A. Oliver, J.A. Cobos, and M. Huemer: "Wireless Charging System Applying Phase-Shift Amplitude Control to Maximize Efficiency and Extractable Power", IEEE Trans. on Pow. El., Vol.30, No.11, pp.6338-6348 (2015)

(22) W.X. Zhong and S.Y.R. Hui: "Maximum energy efficiency tracking for wireless power transfer systems", IEEE Trans. on Pow. El., Vol.30, No.7, pp.4025-4034 (2015)

(23) W.X. Zhong and S.Y.R. Hui: "Charging Time Control of Wireless Power Transfer System Without Using Mutual Coupling Information and Wireless Communication System", IEEE Trans. on Ind. El., Vol.64, No.1, pp.228-235 (2017)

(24) F. Musavi, W. Eberle, and W.G. Dunford: "A High-Performance SinglePhase Bridgeless Interleaved PFC Converter for Plug-in Hybrid Electric Vehicle Battery Chargers", IEEE Trans. on Ind. Appl., Vol.47, No.4, pp.1833$1843(2011)$

(25) G. Lovison, M. Sato, T. Imura, and Y. Hori: "Secondary-side-only simultaneous power and efficiency control for two converters in wireless power transfer system", Proc. 41st Ann. Conf. of the IEEE Ind. El. Soc. (IECON), pp.4824-4829 (2015)

(26) M. Kato, T. Imura, and Y. Hori: "Study on maximize efficiency by secondary side control using DC-DC converter in wireless power transfer via magnetic resonant coupling", in El. Veh. Symp. and Exhib. (EVS27), pp.1-5 (2013)

(27) K. Hata, T. Imura, and Y. Hori: "Maximum Efficiency Control of Wireless Power Transfer Considering Dynamics of DC-DC Converter for Moving Electric Vehicles", in IEEE Appl. Pow. El. Conf. and Expo. (APEC), pp.3301-3306 (2015)

(28) T. Hiramatsu, X. Huang, T. Imura, and Y. Hori: "Wireless Charging Power Control for HESS Through Receiver Side Voltage Control”, in IEEE Appl. Pow. El. Conf. and Expo. (APEC), pp.1614-1619 (2015)

(29) D. Gunji, T. Imura, and H. Fujimoto: "Stability analysis of constant power load and load voltage control method for Wireless In-Wheel Motor", in Proc. 9th Int. Conf. on Pow. El. and ECCE Asia (ICPE-ECCE Asia), pp.1944-1949 (2015)

(30) D. Kobayashi, T. Imura, and Y. Hori: "Real-time coupling coefficient estimation and maximum efficiency control on dynamic wireless power transfer for electric vehicles", in IEEE PELS Workshop on Emerg. Tech.: Wireless Pow. (WoW), pp.1-6 (2015)

(31) "General rules for equipment using high frequency waves: Japanese Electromagnetic Wave Standard", last adjourned in August (2016) (in Japanese)

(32) "Miscellaneous rules for equipment using high frequency waves: Japanese Electromagnetic Wave Standard", last adjourned in August (2016) (in Japanese)

Giorgio Lovison (Student Member) received both the B.S. and M.S.

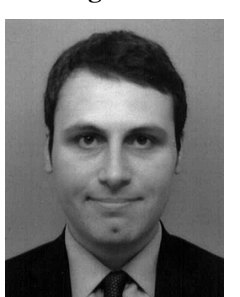
degrees in electrical engineering at the University of Padova, Italy, in 2011 and 2014, respectively. He is currently working towards the Ph.D. degree at The University of Tokyo, Japan. His research interests include wireless power transfer, electric vehicles, and power electronics. He is a student member of IEEE and the Institute of Electrical Engineers of Japan.
Daita Kobayashi (Non-member) received his B.S. degree in applied

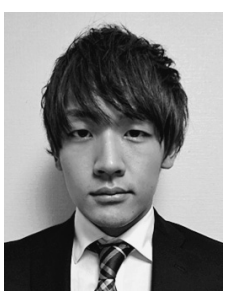
physics from Tokyo University of Science in 2014. He is currently working toward a M.S. degree at the Graduate School of Frontier Sciences with the University of Tokyo. His research interests are mainly on converter control for wireless power transfer via magnetic resonant coupling.

Motoki Sato (Member) received the M.E. degree from Nagaoka Uni-

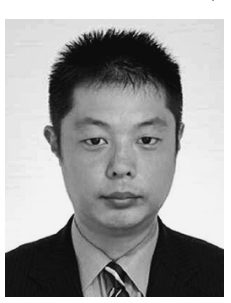
versity of Technology, Japan, in 2005 and the Ph.D. degree from the Graduate School of Frontier Sciences, The University of Tokyo, Japan in 2016. He has been working at Toyo Denki Seizo K.K. as researcher since 2005. His main research interest is power electronics. He is a member of the Institute of Electrical Engineers of Japan.

Takehiro Imura (Member) received the B.S. degree in electrical

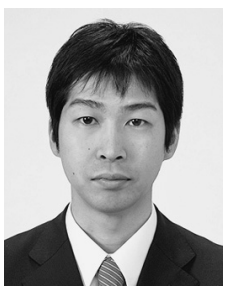
and electronics engineering from Sophia University, Japan, in 2005, and the M.E. degree in electronic engineering and D.Eng. degree in electrical engineering from The University of Tokyo, Japan, in 2007 and 2010, respectively. He is currently a Project Lecturer with the Department of Electrical Engineering, Faculty of Engineering, The University of Tokyo. His main research interest is wireless power transfer coil design, circuit topology and optimization. $\mathrm{He}$ is a member of IEEE, Institute of Electrical Engineers of Japan, Society of Automotive Engineers of Japan and the Institute of Electronics, Information and Communication Engineers.

Yoichi Hori (Fellow) received the B.S., M.S., and Ph.D. degrees in

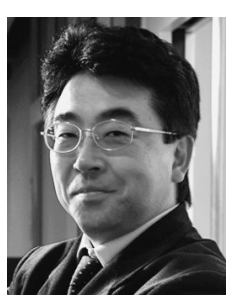
electrical engineering from The University of Tokyo, Tokyo, Japan, in 1978, 1980, and 1983, respectively. In 1983, he joined the Department of Electrical Engineering, The University of Tokyo, as a Research Associate. He later became an Assistant Professor, an Associate Professor, and, in 2000, a Professor at the same university. In 2002, he moved to the Institute of Industrial Science as a Professor in the Information and System Division, and in 2008, to the Department of Advanced Energy, Graduate School of Frontier Sciences, The University of Tokyo. From 1991 to 1992, he was a Visiting Researcher at the University of California at Berkeley. His research fields are control theory and its industrial applications to motion control, mechatronics, robotics, electric vehicles, and wireless power transfer. He has been the Treasurer of the IEEE Japan Council and Tokyo Section since 2001. He was the winner of the Best Transactions Paper Award from the IEEE Transactions on Industrial Electronics in 1993 and 2001, of the 2000 Best Transactions Paper Award from the Institute of Electrical Engineers of Japan (IEEJ), and of the 2011 Achievement Award of the IEEJ. He is an AdCom member of the IEEE Industrial Electronics Society. He is also a member of the Society of Instrument and Control Engineers, Robotics Society of Japan, Japan Society of Mechanical Engineers, and the Society of Automotive Engineers of Japan. $\mathrm{He}$ is the Past President of the Industry Applications Society of the IEEJ, the President of the Capacitors Forum, and the Chairman of the Motor Technology Symposium of the Japan Management Association and the Director of Technological Development of the Society of Automotive Engineers of Japan. 